\title{
Amputee Mobility Predictor-Bilateral: A performance-based measure of mobility for people with bilateral lower-limb loss
}

\author{
Michele A. Raya, PhD, PT, SCS, ATC; ${ }^{1}$ Robert S. Gailey, PhD, PT; ${ }^{1-2^{*}}$ Ignacio A. Gaunaurd, PhD, MSPT; $^{2}$

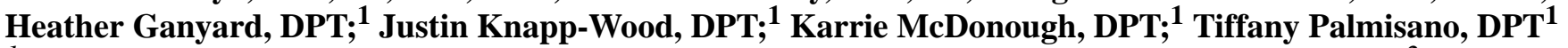 \\ ${ }^{1}$ Department of Physical Therapy, Miller School of Medicine, University of Miami, Coral Gables, FL; ${ }^{2}$ Functional \\ Outcomes Research and Evaluation Center, Miami Department of Veterans Affairs Healthcare System, Miami, FL
}

\begin{abstract}
The Amputee Mobility Predictor (AMP) is an outcome measure designed to assess mobility and functional capabilities for people with unilateral lower-limb amputation. No comparable measure exists for those with bilateral lower-limb amputation (BLLA). The purpose of this study was to examine the utility of the AMP-Bilateral (AMP-B) to measure the ability to perform functional tasks related to participation in advanced skill activities in those with BLLA and to determine whether AMP-B scores correlated with 6-minute walk test (6MWT) performance. Twenty-six male servicemembers (SMs) completed the study: 12 with bilateral transtibial amputation (BTTA), 7 with bilateral transfemoral amputation (BTFA), and 7 with combination transtibial and transfemoral amputation (TTA/TFA). Significant differences existed between the AMPB scores $(p<0.001)$, AMP scores $(p<0.001)$, and 6MWT distance $(p<0.05)$ for SMs with BTTA and TTA/TFA and SMs with BTTA and BTFA but not between those with BTFA and TTA/TFA. Scoring of five AMP items was modified because they necessitate at least one intact knee joint to generate the necessary torque requirements to perform the activity without upper-limb assistance. Minor modifications in scoring of the AMP do not alter total score and allow clinicians to determine the mobility and functional capabilities of SMs with BTFA and TFA/TTA.
\end{abstract}

Key words: 6-minute walk test, Amputee Mobility Predictor, Amputee Mobility Predictor-Bilateral, bilateral amputation, mobility, New Injury Severity Score, outcome measure, servicemembers, traumatic amputation, veterans.

\section{INTRODUCTION}

Approximately 185,000 amputations occur annually in the United States [1]. Although the actual incidence of bilateral amputation has not been reported, it has been established that 30 to 50 percent of people who have an amputation related to diabetes will undergo amputation of the contralateral limb 3 to $5 \mathrm{yr}$ after the primary amputation [2-3]. Moreover, with the increase in world conflict over the past decade with Operation Iraqi Freedom/Operation Enduring Freedom, coupled with medical advancements and improved emergency medical technician

Abbreviations: $6 \mathrm{MWT}=6$-minute walk test, AIS $=$ Abbreviated Injury Scale, AMP = Amputee Mobility Predictor, AMP$\mathrm{B}=\mathrm{AMP}-\mathrm{Bilateral}, \mathrm{ANOVA}=$ analysis of variance, $\mathrm{BLLA}=$ bilateral lower-limb amputation, BTFA = bilateral transfemoral amputation, BTTA = bilateral transtibial amputation, IRB = Institutional Review Board, NISS = New Injury Severity Score, SF-36 = 36-Item Short Form Health Survey, SM = servicemember, TTA/TFA = combination transtibial/transfemoral amputation, WRAMC = Walter Reed Army Medical Center.

*Address all correspondence to Robert S. Gailey, PhD, PT; Department of Physical Therapy, Miller School of Medicine, University of Miami, 5915 Ponce de Leon Blvd, Plummer Building, 5th floor, Coral Gables, FL 33146; 305-2844535; fax: 305-378-4107. Email: rgailey@miami.edu http://dx.doi.org/10.1682/JRRD.2012.05.0097 
services domestically and abroad, the number of individuals surviving with traumatic bilateral lower-limb amputation (BLLA) has increased [4-5]. As of September 2010, of the 1,073 servicemembers (SMs) who have lost limbs, 261 (24\%) have lost multiple limbs. This subpopulation of people with amputation is largely younger and higher functioning than previous generations [5].

Because people with bilateral limb loss are few in number, limited in function, and often not considered prosthetic candidates, a void exists within the literature quantifying or assessing activity and participation restrictions within this group of individuals [6]. The ability to provide an accurate performance-based measurement tool able to quantify impairments of body function that may possibly lead to further activity limitation and participation restrictions is a key step in providing clinicians with tools with which to obtain baseline information, assess progress, and inform treatment planning in people with BLLA.

Functional outcome measures can be classified as either self-report, professional report, or performancebased instruments. Outcome measures for people with lower-limb amputation should have the ability to determine current functional capabilities, inform evidencebased decisions regarding treatment, and potentially predict future levels of function. Functional assessment instruments used with the unilateral lower-limb amputee population have not been validated to assess people with BLLAs [7-10]. For example, the 36-Item Short Form Health Survey (SF-36) has been used as a subjective outcome measure examining quality of life in people with amputation. This tool is self-report and was originally targeted toward the general population. Dougherty performed a study using the SF-36 specifically with BLLA and concluded that the only dimension statistically significant to change is the physical functioning dimension [7]. Another study revealed that, when comparing people with lower-limb amputation with a nondisabled population with intact limbs, the SF-36 does not reflect significant activity limitations or deficits in participation in any of its dimensions [8]. Generic, nonamputation-specific measures of function and quality of life are inappropriate for people with lower-limb amputation. Professional report and self-report instruments such as the Functional Independence Measure, Amputee Activity Survey, SF36, Prosthetic Evaluation Questionnaire, and Orthotics and Prosthetics User's Survey focus on people with unilateral amputation and levels of dependency, are designed to be used in an outpatient setting, and do not address quality of performance because the therapist does not directly observe the performance of the tasks [11-12]. The preferred instruments of function are performance based, where the person must demonstrate ability and not simply self-assess. The Amputee Mobility Predictor (AMP) is an objective performance instrument designed to assess the mobility of people with lower-limb amputation prior to prosthetic fitting and predict function following prosthetic prescription. The initial reliability testing of the AMP included 6 subjects with BLLA and 18 subjects with unilateral lower-limb amputation; however, the AMP was never designed specifically for or validated in people with BLLAs because it was not intended for use with this population [13].

The 6-minute walk test (6MWT) is a widely accepted performance tool used to measure walking capacity in nearly all populations, including people with amputation. It has been shown to have high test-retest reliability and is a practical predictor of community ambulation ability [14]. The 6MWT has been consistently referred to as a gold standard test and shown to correlate with other functional outcome measures in the amputee population [10,13-15]. When compared with the AMP, it was found to have moderate to high correlation in people with lowerlimb amputation [13] but has never been tested for those with BLLA.

Current measures of functional mobility in the amputee population share the same limitation; they have not been validated with BLLA primarily because a cohort with capabilities at higher functional levels is very difficult to recruit. Additionally, bilateral transfemoral amputation (BTFA) results in the loss of bilateral ankle and knee power and postural stabilization, which alters joint movement, resulting in compensatory strategies for balance, mobility, and functional tasks. Compensatory strategies demanded the development of an instrument capable of taking these into consideration when attempting to effectively quantify performance on tasks requiring an intact knee when both anatomical knees are absent. The purpose of this study was to examine the utility of the AMP-Bilateral (AMP-B) to measure the ability to perform functional tasks related to participation in high-level advanced skill activities for those with BLLA and to determine whether AMP-B scores correlated with performance on the 6MWT. 


\section{METHODS}

\section{Study Design}

This cross-sectional, multisite study was conducted at Walter Reed Army Medical Center (WRAMC), Washington, DC; Center for the Intrepid, Brooke Army Medical Center, San Antonio, Texas; and Womack Army Medical Center, Fort Bragg, North Carolina. Prior to signing Institutional Review Board (IRB)-approved informed consent and protected health information, each participant was given eligibility criteria, methodology, confidentiality, and potential risks involved by a research investigator.

\section{Subjects}

Twenty-six male, Active Duty or retired U.S. SMs between the ages of 22 and $40 \mathrm{yr}$ (mean age $28.6 \pm 5.5 \mathrm{yr}$ ) with BLLA resulting from traumatic causes participated in the study (Table 1). SMs consented if they were medically stable with properly fitted prosthesis, demonstrated a minimal level of function as determined by their ability to ambulate a distance of $250 \mathrm{~m}$ in $6 \mathrm{~min}$, and agreed to participate in the study protocol. Subjects were excluded if they had spinal cord injury; upper-limb loss; peripheral nerve injury limiting function; inability to follow commands because of traumatic brain injury; or orthopedic, cardiopulmonary, or contralateral limb injuries limiting mobility or exercise tolerance.

Of the participants, 12 (46\%) had bilateral transtibial amputations (BTTA) and made up the highest proportion of subjects, 7 (27\%) had BTFA, and 7 (27\%) had combination transtibial/transfemoral amputation (TTA/TFA). Mean height was $182.0 \pm 7.7 \mathrm{~cm}$ and mean mass including prosthesis was $91.0 \pm 18.6 \mathrm{~kg}$ (Table 1). Eight (30\%) subjects were retired from the military, eight (30\%) were under Active Duty nondeployed status, two (7\%) were under Active Duty deployed status, and eight (30\%) had completed skilled rehabilitation and were awaiting disposition. No significant difference existed between groups in age, height, and time since initial injury. Significant differences in body mass were found between the groups. Participants with BTTA and TTA/TFA were significantly heavier than those with BTFA, but differences in body mass were not found between participants with BTTA and TTA/TFA.

\section{Study Procedures}

Subjects completed contact information, demographic and anthropometric characteristics, and relevant medical and injury history prior to AMP testing. Injuries were documented by military physical therapists and categorized into respective Abbreviated Injury Scale (AIS) anatomical body regions using coding from the AIS 2005 manual. The New Injury Severity Score (NISS) was given based on the participants' injury history and AIS code. The NISS was calculated using the sum of squares of the three most severe injuries and quantified overall severity of injury to a person [16]. The three most severe injuries sustained were given a classification score of minor (1), moderate (2), serious (3), severe (4), critical (5), or maximal/ currently untreatable (6) [17].

The AMP was administered following the protocol described by Gailey et al. [13]. It is a 20-item assessment tool designed to evaluate the skills required for successful prosthetic ambulation. Items 1 and 2 assess sitting balance; 3 through 7 assess simple mobility: transfers and coming from sit-to-stand; 8 through 13 assess standing balance activities: single-limb stance and pick up object from floor; and 14 through 20 assess components of gait: step length, transverse obstacle, and ascend and descend stairs. All subjects performed the AMP wearing their prostheses. Most items were scored on a 3-point scale with lowest scores correlating with lowest function [18].

Table 1.

Differences in demographic characteristics between servicemembers with different bilateral amputation levels.

\begin{tabular}{|c|c|c|c|c|}
\hline Characteristic & BTTA $(n=12)$ & TTA/TFA $(n=7)$ & $\operatorname{BTFA}(n=7)$ & $p$-Value \\
\hline Age (yr) & $29.8 \pm 5.8(22-40)$ & $28.7 \pm 7.1(22-40)$ & $26.6 \pm 2.8(23-30)$ & 0.50 \\
\hline Height (cm) & $181.9 \pm 6.9(171.5-194.3)$ & $185.4 \pm 9.2(175.3-203.2)$ & $179.1 \pm 7.4$ (170.2-189.2) & 0.30 \\
\hline Body Mass (kg) & $97.7 \pm 20.2(68.0-170.8)$ & $94.9 \pm 13.2(76.6-113.3)$ & $75.0 \pm 9.8(60.7-85.2)$ & $0.02^{*}$ \\
\hline Time Since Initial Injury (yr) & $3.3 \pm 2.7(0.5-10.4)$ & $2.3 \pm 0.8(1.3-3.1)$ & $2.4 \pm 1.1(1.0-4.2)$ & 0.48 \\
\hline
\end{tabular}


When the AMP was performed by those with BLLA, we observed that the scoring of certain items had an unfair bias secondary to mechanical or physical limitations that could never be overcome, regardless of functional level or prosthetic ability. For specific test items, the presence of at least one natural knee is necessary to achieve a maximum score. For example, for a person with two prosthetic knees, the ability to rise out of chair without the use of the upper limbs is regarded as impossible. The AMP score should not penalize the person for use of the upper limbs, which would be necessary for demonstrating functional independence since this lower score would represent a lower level of ability. Five AMP items were modified because intact knee extensors are necessary to perform these tasks and achieve top scores. These five items were ability to rise from a chair, attempts needed to rise from a chair without the use of the upper limbs, balance immediately upon standing measured by accessory movements needed to maintain position, ability to descend into a chair without the use of the upper limbs, and ability to climb up and down stairs without using the upper limbs. All participants with BLLA were retrospectively graded with a new scoring system (AMP-B). See the Appendix for scoring modifications (available online only).

The 6MWT was administered on a level surface. Subjects were instructed to walk at a self-selected pace, without running, for a total time of $6 \mathrm{~min}$. Subjects were allowed to rest and restart if needed during the $6 \mathrm{~min}$; however, time was not stopped. The total distance walked was measured in meters.

\section{Data Analysis}

Data analysis was performed using SAS version 9.1.3 (SAS Institute Inc; Cary, North Carolina). Descriptive sta- tistics on the demographic data, including age, height, body mass, and time since injury by group, were calculated and are shown in Table $\mathbf{1}$.

Analysis of variance (ANOVA) was used to examine significant differences between groups in reference to NISS, AMP, and AMP-B scores; differences between AMP and AMP-B scores; and 6MWT distance. Results are shown in Table 2 . When the results were statistically significant $(p<0.05)$, post hoc Tukey tests were used to perform pairwise comparisons of the groups. Pearson product-moment correlation coefficients were calculated to determine the relationship between NISS scores and 6MWT with AMP and AMP-B scores (Table 3).

\section{RESULTS}

Table 4 describes the type of prosthetic components worn during the testing session by the participants. The majority of participants (85\%) wore J-shaped energy storing and returning feet with or without shock absorption and torque rotators. The majority of participants with either TTA/TFA or BTFA wore microprocessor knee units (71\%).

The ANOVA results demonstrated a significant difference between all three groups for each variable represented in Table $2(p<0.05)$. Post hoc analysis found the following: The NISS scores differed between all three groups, indicating that participants with BTFA had greater injury severity than those with TTA/TFA and BTTA, and those with TTA/TFA had greater injury severity than those with BTTA. AMP scores were different between all three

Table 2.

Outcome measure between servicemembers with different bilateral amputation levels.

\begin{tabular}{|c|c|c|c|c|}
\hline Outcome Measure & BTTA $(n=12)$ & TTA/TFA $(n=7)$ & BTFA $(n=7)$ & $p$-Value \\
\hline$\overline{\text { NISS*†‡ }}$ & $21.4 \pm 3.1(18-27)$ & $29.7 \pm 4.2(25-34)$ & $35.4 \pm 2.9(33-41)$ & $<0.001$ \\
\hline AMP Score ${ }^{* \dagger \ddagger}$ & $43.3 \pm 2.2(39-46)$ & $38.7 \pm 1.9(36-41)$ & $35.3 \pm 2.6(32-39)$ & $<0.001$ \\
\hline AMP-B Score ${ }^{\dagger \ddagger}$ & $44.3 \pm 1.1(43-46)$ & $41.4 \pm 1.7(39-44)$ & $39.0 \pm 2.8(35-43)$ & $<0.001$ \\
\hline $\begin{array}{l}\text { Difference between AMP } \\
\text { and AMP-B Score }\end{array}$ & $1.0 \pm 1.6(0-4)$ & $2.7 \pm 0.8(2-4)$ & $3.7 \pm 0.5(3-4)$ & $<0.001$ \\
\hline $6 \mathrm{MWT}(\mathrm{m})^{*}$ & $576.1 \pm 75.1(482.4-718.3)$ & $503.1 \pm 88.7$ (323.9-614.6) & $451.6 \pm 141.2(264.1-645.1)$ & 0.05 \\
\hline
\end{tabular}


Table 3.

Relationship between New Injury Severity Score (NISS) and 6-minute walk test (6MWT) distance and Amputee Mobility Predictor (AMP) and AMP-Bilateral (AMP-B) for servicemembers with different bilateral amputation levels $(n=26)$. For data shown, all $p<0.001$.

\begin{tabular}{lcc}
\hline $\begin{array}{l}\text { Correlation } \\
\text { Coefficient }\end{array}$ & AMP $(\boldsymbol{n}=\mathbf{2 6})$ & AMP-B $(\boldsymbol{n}=\mathbf{2 6})$ \\
\hline NISS & -0.824 & -0.790 \\
6MWT & 0.603 & 0.603 \\
\hline \hline
\end{tabular}

groups indicating that participants with BTTA scored higher than those with TTA/TFA and BTFA, and those with TTA/TFA scored higher than those with BTFA. AMP-B scores were different between all groups except between participants with BTFA and TTA/TFA. The participants with BTFA and TTA/TFA scores increased the most from the modification in the AMP scoring. Both groups were unable to perform tasks requiring intact knee extensor muscles and required the scoring adjustment. Higher AMP-B scores more accurately reflect functional capabilities and limited the variability in the scores. AMP$\mathrm{B}$ scores were elevated in comparison with AMP scores within all groups $(p<0.001)$. Participants with BTTA had a mean increase of 1.0 points between the AMP-B and AMP scores. Increases of 3.7 and 2.7 points between AMP-B and AMP scores were found in the BTFA and TTA/TFA groups, respectively. The 6MWT distance differed significantly between those with BTTA and BTFA $(p<0.05)$ but not between those with BTTA and TTA/TFA or between those with BTFA and TTA/TFA.

The NISS had a strong negative correlation with AMP and AMP-B scores $(p<0.001)$, indicating that higher AMP and AMP-B scores were related to lower NISS scores. The 6MWT had a moderate strong correlation with the AMP and AMP-B scores, indicating that higher AMP and AMP-B scores were related to greater distance walked in $6 \mathrm{~min}$.
Age, height, and time since initial injury were not significantly correlated with the AMP-B or AMP score, nor were they different between groups at baseline. Body mass of the subjects was a significant predictor of both AMP-B and AMP score $(p<0.01)$. Variations in body mass can be attributed to the different levels of amputation across groups. The NISS, AMP, and AMP-B scores correlated with 6MWT performance.

\section{DISCUSSION}

Five AMP items were modified to retrospectively score participants on the AMP-B. We found that SMs with BTFA were physically unable to perform the two items that required participants to rise from a chair without the use of upper-limb support. Scoring of these items was modified because absence of an intact knee joint diminishes the ability of the knee extensors to generate the necessary torque required to extend the knee and rise from a chair without upper-limb assistance. Without modifications, even an extremely high functioning BTFA would be unable to obtain the maximal AMP score.

In another item that required modifications, participants were instructed to maintain quiet, controlled stance immediately upon standing. We observed that not all SMs with BTFA and TTA/TFA were able to immediately achieve quiet standing without taking small steps to adjust base of support and make minor accommodations for the relationship between the proximal socket, bordering anatomy, and clothing. These compensatory strategies have been related to the transection of the adductor musculature that occurs during amputation leading to femoral abduction bias [19], requiring a wider base of support in standing. The use of a wider base of support during sit-to-stand transfers can also be a result of prosthetic training. Therefore, these immediate adjustments

Table 4.

Prosthetic components used during Amputee Mobility Predictor (AMP) by people with bilateral lower-limb amputation ( $n=26)$.

\begin{tabular}{|c|c|c|c|c|}
\hline \multicolumn{3}{|c|}{ Prosthetic Ankle/Foot Assemblies } & \multicolumn{2}{|c|}{ Prosthetic Knee Units } \\
\hline $\begin{array}{c}\text { J-Shaped ESAR SAT } \\
\text { Foot, } n(\%)\end{array}$ & $\begin{array}{c}\text { J-Shaped ESAR } \\
\text { Foot, } n(\%)\end{array}$ & $\begin{array}{c}\text { Low-Profile ESAR } \\
\text { Foot, } n(\%)\end{array}$ & $\begin{array}{l}\text { Microprocessor, } \\
n(\%)\end{array}$ & $\begin{array}{c}\text { Nonmicroprocessor, } \\
n(\%)\end{array}$ \\
\hline $13(54)$ & $4(15)$ & $8(31)$ & $10(71)$ & $4(29)$ \\
\hline \multicolumn{5}{|c|}{$\begin{array}{l}\text { Note: One participant with combination transtibial/transfemoral amputation wore J-Shaped ESAR Foot with SAT on transtibial side and low-profile ESAR Foot on } \\
\text { transfemoral side. Otherwise, all participants wore same kind of foot for both limbs. All participants with BTFA used same category of prosthetic knee unit for both } \\
\text { limbs. Össur Total Knee } 1900 \text { was only knee used by participants that lacks yield-rate control. } \\
\text { ESAR = energy storing and returning, SAT = shock absorption and/or torque rotator. }\end{array}$} \\
\hline
\end{tabular}


are indicative of functional control strategies unique to those with BTFA. Modifications of the AMP that allow for controlled adjustments during immediate standing will enable the instrument to more appropriately assess balance and standing strategies.

Similar issues arose in an item requiring participants to control descent into a chair without upper-limb support. Upper-limb assistance is essential for those with BTFA and TTA/TFA to control descent into a chair because this task requires an eccentric contraction of the knee extensors to control knee flexion. Individuals with BTFA lack knee extensors that cross an intact knee joint, rendering them unable to control knee flexion during descent without additional support. Many prosthetic knee designs, but not all, permit up to $30^{\circ}$ of yield-rate control with the intention of slowing knee flexion or the person's descent when sitting in a chair. For an individual with BTFA who is fitted with prosthetic knees with yield-rate control, proper technique may be taught for stand-to-sit transfer where controlled prosthetic knee flexion is used until the hands reach the arms of the chair, with the remainder of controlled descent made possible by the upper limbs. Therefore, one with BTFA who performs independent controlled decent into a chair with upperlimb support has demonstrated mastery of the task and should be credited the maximum score.

Lastly, modifications were required to the item that scored participants' ability to ascend/descend stairs. Those with BTFA were unable to perform this task without upper-limb support because of the absence of intact knee extensors, as previously discussed. Instead, they used a variety of strategies involving the upper limbs. Safe, independent ascent and descent of stairs with upper-limb support should be awarded the maximum score. Although literature examining predictive measures of functional mobility for those with BLLA is limited, our findings are consistent with previous studies that state the presence of at least one intact knee is key to maximizing function [20].

BTFA results in the loss of bilateral ankle and knee power and postural stabilization, which alters joint movement, resulting in compensatory strategies for balance, mobility, and functional tasks. Compensatory strategies demanded the development of the AMP-B to effectively quantify performance on tasks requiring an intact knee when both anatomical knees are absent. The AMP-B scores of participants increased in comparison with their AMP scores after modifications were made in the scoring proto$\mathrm{col}$. The SMs with BTTA improved the least from the presence of both anatomical knees, which permitted normal hip and knee movement and allowed for typical scoring on the AMP. The SMs with TTA/TFA improved slightly from the presence of one intact knee, thereby allowing knee extensor function in one limb. The SMs with BTFA benefitted the most because of the scoring adjustments to the AMP designed to accommodate for the absence of anatomical knees and the resultant lack of muscle power that prevented them from performing the aforementioned tasks. Modifications to the AMP created a more sensitive tool for determining functional capabilities in those with BTFA and TTA/TFA. When comparing AMP-B scores, the BTTA group differed significantly from the other groups. No differences were found between the BTFA and TTA/TFA groups. This confirms that BTTA did not necessitate scoring modifications, whereas both the BTFA and TTA/TFA required a scoring adjustment to be comparable to other amputee groups being administered the AMP.

With the modified AMP scoring, BTFA and TTA/ TFA were no longer penalized for using adaptive mobility strategies such as using upper-limb assistance when rising from a chair or taking a step during immediate standing. The functional capabilities of those with BTFA and TTA/ TFA were not as limited as standard AMP scores suggested. The AMP-B provides a more accurate assessment of the functional capabilities of SMs with BLLA.

The NISS scores had a very strong, negative correlation with AMP-B scores. The more involved the limb loss, the greater the injury severity or higher NISS score, resulting in a very strong correlation between NISS score, level of amputation, and AMP-B score. This further validates the adjustments in AMP scores for those with TTA/TFA and BTFA.

A strong correlation exists between the AMP-B and the 6MWT, allowing us to predict those with higher scores on the AMP-B will have greater overall functional mobility. The AMP was previously validated as a predictive tool of 6MWT performance. Modifications to the AMP in development of the AMP-B did not affect its capacity to predict mobility.

The group with two anatomical knees (BTTA) walked further than both the BTFA and TTA/TFA groups. Likewise, those with even a single anatomical knee demonstrated increase walking distance over those with no knees (BTFA). The 6MWT distance was found to differ significantly only between the BTTA and BTFA groups, indicating the AMP-B may be more sensitive than the 6MWT, where differences were found between the BTTA, BTFA, and the TTA/TFA groups. The 6MWT, a measure of aerobic capacity, requires the knee to function 
as a simple hinge, as during bipedal ambulation. The AMP-B, however, requires the ability to rise from a chair, balance, step over an obstacle, and negotiate stairs, all necessitating the knee to execute a variety of complex tasks requiring greater demands on the joint and surrounding musculature.

Although this study was designed to target higher functioning individuals with traumatic bilateral amputation, future work should include people with bilateral amputation who had lower-level AMP scores. Despite the overall small sample size, this is one of the largest performance-based outcomes studies conducted to date measuring functional mobility for those with BLLA. Future work should enable clinicians to determine the capabilities of this population with an instrument that allows them to measure an obtainable goal. When patients have difficulty performing a specific task (i.e., standing from a chair), a treatment prescription and training technique may be applied, enabling the patient and clinician to clearly assess where they are currently and when they have reached maximum independence. Again, the techniques using assistive devices and chair arms for standing are completely different for this population than those with two knees.

\section{CONCLUSIONS}

The AMP is used to determine functional mobility of people with all levels of unilateral lower-limb amputation. Recent world events have resulted in an increase of individuals with BLLA and therefore a need for an instrument to determine their participation restrictions and abilities. Specific tasks within the AMP may have unintentional bias for people with bilateral limb loss, in particular those with BTFA because of the absence of intact knees. We determined that minor modifications in scoring of the AMP, without altering the total score, allow clinicians to more accurately determine the participation abilities of this population at higher functioning levels. No modifications are necessary for people with BTTA. In addition, the AMP-B and the AMP have a strong correlation with the 6MWT in the higher functioning people with amputation.

\section{ACKNOWLEDGMENTS}

Author Contributions:

Study concept and design: M. A. Raya, R. S. Gailey.
Acquisition of data: M. A. Raya.

Analysis and interpretation of data: M. A. Raya, R. S. Gailey, I. A. Gaunaurd.

Drafting of manuscript: M. A. Raya, R. S. Gailey, I. A. Gaunaurd, H. Ganyard, J. Knapp-Wood, K. McDonough, T. Palmisano.

Critical revision of manuscript of important intellectual content: M. A. Raya, R. S. Gailey, I. A. Gaunaurd.

Statistical analysis: I. A. Gaunaurd.

Study supervision: M. A. Raya, R. S. Gailey.

Financial Disclosures: The authors have declared that no competing interests exist.

Funding/Support: This material is based on work supported by the Military Amputee Research Program and the Telemedicine and Advanced Technology Research Center (Prime Award No.

W81XWH-06-2-0073), administered by the Henry M. Jackson Foundation for the Advancement of Military Medicine Inc and the South

Florida Veterans Affairs Foundation for Research and Education Inc.

Additional Contributions: The authors would like to thank Alison A. Linberg, DPT, ATC; MAJ (Ret) Stuart M. Campbell, MPT; LTC Daniel M. Jayne, MPT, OCS; Kathryn E. Roach, PhD, PT; COL (Ret) Charles Scoville, PT, PhD; COL (Ret) Rebecca Hooper, PhD, PT; COL (Ret)

Paul D. Stoneman, PhD, MPT, DPT, OCS, SCS; Carlos GomezOrozco; Justin Z. Laferrier, PhD, MSPT, OCS, SCS, ATP, CSCS; John R. Fergason, CPO; Peter D. Harsch, CP; CAPT (Ret) Kathy Goldberg, MSPT; Nico Marcolongo; Matt Berliner, DPT; Jen Berrios, DPT; Todd Bowen, DPT; Sarah Carballo, DPT; Marcos Davy, DPT; Kayla Felderhoff, DPT; Erica Gagne, DPT; Erick Harada, DPT; Carolyn Lindsay, DPT; Emily Lo, DPT; Megan Mannikko, DPT; Patrick G. Manrique, DPT; Karrie McDonough, DPT; Daniel G. Muller, DPT; Jeremiah Randall, DPT, ATC; Emily Roberts, DPT; Jarrod Schechla, DPT; Catherine Stewart, DPT; Adam Stivala, DPT; and Christen Tucker, DPT, for their dedication and countless hours of work contributed to make this project a success. The authors also thank the staff at the Funk Physical Fitness Center and Womack Army Medical Center in Fort Bragg; Center for the Intrepid, Brooke Army Medical Center; Military Advanced Training Center, WRAMC; Amputee Care, Prosthetics, and Rehabilitation Department, Naval Medical Center San Diego; Research Department, Miami Department of Veterans Affairs Healthcare System; South Florida Veterans Affairs Foundation for Research and Education; and Challenged Athletes. Ms. Ganyard is now with Sibley Memorial Hospital, Washington, DC. Mr. Knapp-Wood is now with Bechtel Physical Therapy, Los Angeles, California. Ms. McDonough is now with Arthritis and Rheumatism Associates, P. C., Washington, DC. Ms. Palmisano is now with South Texas Surgical Hospital, Corpus Cristi, Texas. The Amputee Mobility-Bilateral: Copyright 2009 Advanced Rehabilitation Therapy Inc, Miami, Florida. Reprinted with permission.

Institutional Review: This study was approved by the IRB at Womack Army Medical Center, WRAMC Department of Clinical Investigation, the WRAMC Human Use Committee (HUC), Brooke Army Department of Clinical Investigation, the Brooke Army Medical Center HUC, the Army Clinical Investigation Regulatory Office, and Human Studies Subcommittee at the Miami Department of Veterans Affairs Healthcare System.

Participant Follow-Up: The authors plan to inform participants of the publication of this study. 


\section{REFERENCES}

1. Amputee Coalition [Internet]. Manassas (VA): 2013 [cited $2010 \mathrm{Jul}$ 27]. Available from: http://www.amputee-coalition.org/.

2. Izumi Y, Lee S, Satterfield K, Harkless L. Risk of reamputation in diabetic patients stratified by limb and level of amputation: a 10-year observation. Diabetes Care. 2006; 29(3):566-70. [PMID:16505507]

http://dx.doi.org/10.2337/diacare.29.03.06.dc05-1992

3. Mazet R, Schiller FJ, Dunn OJ, Neufeld AJ. The influence of prosthesis wearing on the health of the geriatric amputee. Project 431, California Office of Vocational Rehabilitation, March 1963.

4. Reiber GE, Smith DG. VA paradigm shift in care of veterans with limb loss. J Rehabil Res Dev. 2010;47(4):vii-x. PMID:20803394] http://dx.doi.org/10.1682/JRRD.2010.03.0030

5. Dougherty PJ, McFarland LV, Smith DG, Esquenazi A, Blake DJ, Reiber GE. Multiple traumatic limb loss: A comparison of Vietnam veterans to OIF/OEF servicemembers. J Rehabil Res Dev. 2010;47(4):333-48. [PMID:20803402] http://dx.doi.org/10.1682/JRRD.2009.04.0043

6. Department of Health and Human Services. Medicare region $\mathrm{C}$ durable medical equipment regional carrier, supplier update workshops. Washington (DC): Department of Health and Human Services; 1995.

7. Dougherty PJ. Long-term follow-up study of bilateral above-the-knee amputees from the Vietnam War. J Bone Joint Surg Am. 1999;81(10):1384-90. [PMID:10535588]

8. Taghipour H, Moharamzad Y, Mafi AR, Amini A, Naghizadeh MM, Soroush MR, Namavari A. Quality of life among veterans with war-related unilateral lower extremity amputation: A long-term survey in a prosthesis center in Iran. J Orthop Trauma. 2009;23(7):525-30. [PMID:19633463] http://dx.doi.org/10.1097/BOT.0b013e3181a10241

9. Abramson J, Abramson Z. Survey methods in community medicine. 5th ed. Edinburgh (UK): Churchill Livingstone; 1999.

10. Condie E, Scott H, Treweek S. Lower limb prosthetic outcome measures: A review of the literature 1995 to 2005. J Prosthet Orthot. 2006;18(6):P13-P45. http://dx.doi.org/10.1097/00008526-200601001-00004

11. Leung EC, Rush PJ, Devlin M. Predicting prosthetic rehabilitation outcome in lower limb amputee patients with the functional independence measure. Arch Phys Med Rehabil. 1996;77(6):605-8. [PMID:8831480] http://dx.doi.org/10.1016/S0003-9993(96)90303-2

12. Panesar BS, Morrison P, Hunter J. A comparison of three measures of progress in early lower limb amputee rehabilitation. Clin Rehabil. 2001;15(2):157-71. [PMID:11330761] http://dx.doi.org/10.1191/026921501669259476
13. Gailey RS, Roach KE, Applegate EB, Cho B, Cunniffe B, Licht S, Maguire M, Nash MS. The amputee mobility predictor: An instrument to assess determinants of the lowerlimb amputee's ability to ambulate. Arch Phys Med Rehabil. 2002;83(5):613-27. [PMID:11994800]

14. Lin SJ, Bose NH. Six-minute walk test in persons with transtibial amputation. Arch Phys Med Rehabil. 2008; 89(12):2354-59. [PMID:18976979] http://dx.doi.org/10.1016/j.apmr.2008.05.021

15. Gailey R. Predictive outcome measures versus functional outcome measures in the lower limb amputee. J Prosthet Orthot. 2006;18(6):P51-P60.

http://dx.doi.org/10.1097/00008526-200601001-00006

16. Osler T, Baker SP, Long W. A modification of the injury severity score that both improves accuracy and simplifies scoring. J Trauma. 1997;43(6):922-25, discussion 925-26. [PMID:9420106] http://dx.doi.org/10.1097/00005373-199712000-00009

17. Gennarelli T, Wodzin E. Abbreviated Injury Scale (AIS). Barrington (IL): Association for the Advancement of Automotive Medicine; 2005.

18. Raya MA, Gailey RS, Fiebert IM, Roach KE. Impairment variables predicting activity limitation in individuals with lower limb amputation. Prosthet Orthot Int. 2010;34(1):73-84. [PMID:20196689] http://dx.doi.org/10.3109/03093640903585008

19. Gottschalk F. [The biomechanics of transfemoral amputations]. Orthopädie-Technik Quarter. 2005;3(6):164-69. German.

20. Volpicelli LJ, Chambers RB, Wagner FW Jr. Ambulation levels of bilateral lower-extremity amputees. Analysis of one hundred and three cases. J Bone Joint Surg Am. 1983; 65(5):599-605. [PMID:6853564]

Submitted for publication May 30, 2012. Accepted in revised form September 26, 2012.

This article and any supplementary material should be cited as follows:

Raya MA, Gailey RS, Gaunaurd IA, Ganyard H, KnappWood J, McDonough K, Palmisano T. Amputee Mobility Predictor-Bilateral: A performance-based measure of mobility for people with bilateral lower-limb loss. J Rehabil Res Dev. 2013;50(7):961-68.

http://dx.doi.org/10.1682/JRRD.2012.05.0097

ResearcherID/ORCID: Robert S. Gailey, PhD, PT: I-34582013; Ignacio A. Gaunaurd, PhD, MSPT: I-3490-2013

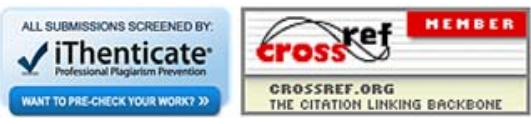

